\title{
TONGUE DIAGNOSIS OF TRADITIONAL CHINESE MEDICINE FOR RHEUMATOID
}

ARTHRITIS

\section{Lun-Chien Lo ${ }^{1,3}$, Chia-Yun Chen ${ }^{1}$, John Y. Chiang ${ }^{2}$, Tsung-Lin Cheng ${ }^{3}$, Hong-Jen Lin ${ }^{4}$, Hen-Hong Chang, ${ }^{5}$}

${ }^{1}$ Department of Chinese Medicine, Changhua Christian Hospital, Taiwan. ${ }^{2}$ Department of Computer Science and Engineering, National Sun Yat-sen University, Taiwan. ${ }^{3}$ Department of Mathematics and Institute of Statistics and Information Science, National Changhua University of Education, Taiwan. ${ }^{4}$ Department of Traditional Chinese Medicine, China Medical University Hospital, Taiwan. ${ }^{5}$ Chang Gung Memorial Hospital and Chang Gung University College of Medicine, Taiwan.

*E-mail: $137877 @$ cch.org.tw

\begin{abstract}
Rheumatoid arthritis (RA) is a chronic, systemic autoimmune disease with unknown aetiology that causes the immune system to attack the joints (synoviums), leading to chronic inflammation. According to the traditional Chinese medicine (TCM), RA falls into the category of Impediment disease ("Bi” syndrome), that is, poor circulation of qi and blood (stasis). Tongue diagnosis is an important method of TCM to detect blood stasis. In this study, 74 RA patients, meeting the pre-set criteria, were recruited via rheumatology outpatient clinic and examined by experienced rheumatology physicians. Two images - one of the tongue and the other, sublingual vessels - of the same patient were taken by a Canon digital camera in a darkroom with uniform lighting conditions. Relevant features of the tongue were extracted by utilising image processing techniques. Every tongue was classified into corresponding patterns based on the features identified. The subjects included 62 females and 12 males with an average age of $49.86 \pm 13.81$ years old, an average morbidity period of $4.56 \pm 3.92$ years, an average rheumatoid factor (RF) of $225.3 \pm 373.8 \mathrm{IU} / \mathrm{mL}$ and an average erythrocyte sedimentation rate of (ESR) $40.9 \pm 31.9 \mathrm{~m} / \mathrm{hr}$. According to our study, $86 \%$ of the patients with RA have tongues with sublingual vessels with a width of more than $2.7 \mathrm{~mm}$, a length of more than $3 / 5$ from tongue tipto sublingual caruncle, or a count of sublingual vessels more than 2. Moreover, since RA index is highly correlated with blood stasis in TCM, a logistic regression is conducted to predict the probability of presence of RA using RF and ESR as explanatory variables. Also, the logistic regression analysis of RA with respect to the conventional tongue diagnosis criteria was performed. Based on the aforementioned studies, we concluded that tongue diagnosis is helpful in detecting blood stasis of RA.
\end{abstract}

Key words: TCM, Rheumatoid arthritis, Tongue diagnosis, Rheumatoid factor, Blood stasis

\section{Introduction}

Rheumatoid arthritis (RA) is a chronic, inflammatory disease in which more than 3 peripheral joint areas including the metacarpophalangeal (MCP) joints, proximal interphalangeal (PIP) joints, metatarsophalangeal joints and wrists are involved 
http://dx.doi.org/10.4314/ajtcam.v10i5.24

(Choy and Panayi 2001; Fleming et al. 1976). It usually progresses from periphery to more proximal joints. The symptoms of RA start with insidious pain, stiffness, and swelling of the joints, resulting in erosion of cartilage and bone which would lead to joint deformity (Lee and Weinblatt 2001).

The main biologic markers for RA nowadays are rheumatoid factor (RF), antibodies to citrullinated peptides, erythrocyte sedimentation rate (ESR) and C-reactive protein (CRP) (Otterness 1994; Crowson et al. 2009). Although RF was reported detectable in 70\% 90\% of the RA patients (Shmerling and Delbanco 1991), population-based studies, which include patients with mild RA are found with lower RF-positive rates (26 to 60 percent) (Lichtenstein and Pincus 1991). Many acute phase reactants (APR), e.g., ESR and CRP, are common laboratory tests in inflammation condition, but ESR gains wider acceptance in distinguishing between early RA, osteoarthritis, and SLE (Donald and Ward 1998). Besides, CRP may trigger immune complex and play a role in pathogenesis of rheumatoid arthritis. (Molenaar et al. 2001) ESR and CRP are helpful in monitoring the activity of diseases (Crowson et al. 2009; Cylwik et al. 2010).

The diagnosis of RA is based on the criteria established in 1987. The criteria include the aggregation of clinical history, physical examination, laboratory tests, radiologic findings and exclusion of other diagnoses. (MacGregor 1995) The 1987 American Rheumatism Association criteria are morning stiffness, arthritis of more than 3 joint areas, arthritis of hand joints, symmetric arthritis, rheumatoid nodules, serum rheumatoid factor, and radiographic changes. Other diseases such as reactive arthritis (Toivanen and Toivanen 2004), psoriatic arthritis (Moll and Wright 1973), and Osteoarthritis (OA) must be excluded before the diagnosis of RA is established.

According to TCM theory, RA falls into the category of "Impediment disease" (Sionneau and Gang 1998; Wiseman 1995) (also known as "Bi" syndrome), that is, a group of diseases caused by the invasion of wind, cold, dampness or heat pathogen on the meridian channels (World Health Organization. Regional Office for the Western 2007). The term "Bi" means "blockage." The disease may involve muscles, bones and joints, manifested by local pain, soreness, heaviness, and even articular swelling, stiffness and deformities. (Unschuld 2003) We can diagnose impediment disease by correlation of all four examinations of TCM (inspection, listening and smelling, inquiry, and palpation). Depending upon the inherent characteristics, impediment disease can be classified into three different types: moving, painful and fixed impediments. Moving impediment (wind impediment) is characterised by migratory joint pain. Painful impediment (cold impediment) is when severe joint pain is exaggerated by coldness. On the other hand, fixed impediment (dampness impediment) exhibits fixed joint pain. (Liu 2005)

Tongue diagnosis is an important method in TCM to detect constitution like qi deficiency, yin deficiency, blood stasis, dampness, etc. (Schnorrenberger and Schnorrenberger 2005). In tongue diagnosis, observation of the surface of the tongue is mainly focused on the shape, fur, and body of the tongue. The shape of the tongue can be medium, fat, thin, and tilted. A normal tongue should be one with a medium shape. A fat tongue indicates water toxin, while a thin tongue represents yin vacuity or qi and blood vacuity. In terms of the tongue body, it can be pale, light-coloured, light red, red, crimson, or dark-coloured with bruise dots, fissures, tooth marks, or red dots. A normal tongue exhibits light-red colour. When the tongue is pale, it reflects qi and blood vacuity. When it is red or crimson, it represents repletion heat or yin vacuity fire effulgence. Fissures often appear with malnutrition. On the other hand, tooth marks correspond to qi vacuity or water toxin. The fur of the tongue can be white, yellow, black, slimy, thick, thin, peeling or none at all. Pale fur is considered normal. Thick fur is mapped to bad stomach qi. Peeling fur corresponds to weak stomach qi or stomach yin insufficiency. Yellow fur represents diseases caused by heat. Gray or black fur means toxin deposit or diseases caused by cold. Common body fluid is normal. 
Insufficient or lack of body fluid means yin vacuity. Observation at the back of the tongue is focused on the sublingual collateral vessels, including the length, width, number of branches, solidness, and colour of the back of the tongue. By inspecting these features, a doctor can have an understanding of the patient's qi and blood circulation, and whether organs are functioning normally. Observation of the tongue status starts with the visible abnormality which accordingly becomes the basis for pattern identification/syndrome differentiation. (Maciocia 2004) In recent studies, some researchers noted an unusual appearance of the tongue. (Basak and Jesmajian 2010) Some of them were patients with long standing hyperglycaemia. The appearances of tongue showed severe xerostomia or a coated tongue. (Negrato and Tarzia 2010)

The symptoms of RA were similar to those of the impediment disease. To the best of our knowledge, there was no prominent evidence between these two reported in the past. In order to explore the relations between RA and impediment disease, data obtained from the laboratory and TCM tongue diagnoses are analysed in the following sections. The identification of RA through TCM tongue diagnosis is performed. The effectiveness of linking tongue features with RA is verified through statistical analysis. The relationship between TCM and Western medicine in detecting blood stasis of RA is explored. The new findings hopefully shed light on the differentiation and possible future prognosis and treatment of RA from impediment disease.

\section{Materials and Methods}

\section{Subjects}

In this study, we recruited RA patients who were diagnosed by rheumatology physicians based on 1987 ARA criteria in the TCM internal medicine clinic.

\section{Inclusion Criteria:}

1. Adult patients

2. Patients with confirmed diagnosis of RA

Exclusion criteria

1. Patients under the age of 16

2. Unconscious patients

3. Patients unable to protrude the tongue stably

\section{Methods}

First, the patients completed the general data and recorded the laboratory data including RF, CRP, and ESR. Then, an experienced TCM doctor performed four examinations of TCM, i.e., inspection, listening and smelling, inquiry, and palpation, on the patients. Special attention was focused on the inspection of tongue. Images of tongue and sublingual vessels, as shown in Figs. 3 and 4, were taken in a standardised environment by using a digital camera (Canon EOS400D) with an auxiliary light source (Brite Vision ${ }^{\circledR}$ LED ring light) under a uniform temperature $\left(25^{\circ} \mathrm{C}\right)$ and humidity $(60 \%)$. Every image of the tongue and sublingual vessels was inspected by three experienced TCM doctors, respectively. Features, including size, shape, colour, moisture and coating, of the tongue were observed and manually recorded. 
http://dx.doi.org/10.4314/ajtcam.v10i5.24

\section{Statistical Analysis}

A generalised linear model for each of the three RA indexes - RF, ESR and CRP - with respect to the conventional criteria in tongue diagnosis was performed. The Statistical software, Minitab, provides not only the estimations of regression but also the ANOVA output. The linear model employed is listed below:

$$
Y=a+b_{1} Z_{1}+b_{2} Z_{2}+b_{3} Z_{3}
$$

where $Y$ denotes the RF, ESP or CRP index of the individuals, $Z_{1}$ stands for the indicator of whether an individual is diagnosed as being with purple tongue (PT), $Z_{2}$ is the indicator of whether an individual is diagnosed as being with stasis macules of tongue (SMT), and $Z_{3}$ indicates that an individual is diagnosed as being with sublingual blood stasis pattern (SBSP). Note that $Z_{1}, Z_{2}$ and $Z_{3}$ assume only binary values 0 and 1 , and this observation leads us to utilising the generalised linear model (1) as the means of analysis.

\section{Results}

We recruited seventy-eight patients for the study, and successfully completed a total of seventy-four cases. The reason for patients failing the study was that they could not complete the laboratory data. Participants ranged from 16 to 74 years old at an average age of $49.86 \pm 13.81$ years old. Among them, 62 were females and 12 were males. The average RF was 225.3 $\pm 373.8 \mathrm{IU} / \mathrm{mL}$, the average ESR $40.9 \pm 31.9 \mathrm{~mm} / \mathrm{hr}$., and the average CRP was $1.21 \pm 1.97 \mathrm{mg} / \mathrm{dl}$ (with confidence level of $95 \%$ ). The probability of correct diagnosis, measured by the conventional criteria for determining the presence of the blood stasis including purple tongue, stasis macule on the tongue, and sublingual blood stasis pattern is $93.24 \%$. The general data and laboratory data of the patients are listed in Tables 1 and 2 respectively.

From the summary of general data (Table 1), laboratory data (Table 2) and ratio of patients with different TCM tongue characteristics (Table 3), we could see that the ratio of blood stasis pattern is $86.49 \%$, while that of sublingual blood stasis pattern is $86.49 \%$. The strong correlation between RA and blood statis demonstrates that RA can be reliably identified through the inspection of tongue by following the TCM theory of impediment disease.

Table 1: Characteristics of recruited subjects

\begin{tabular}{lc}
\hline & Mean \pm SD \\
\hline Age(year) & $49.86 \pm 13.81$ \\
Morbidity time(year) & $4.56 \pm 3.92$ \\
\hline Cases & \\
\hline Male & 12 \\
$\quad$ Female & \\
\hline
\end{tabular}

Table 2: Laboratory data of RA patients

\begin{tabular}{lc}
\hline Laboratory data & Mean \pm SD \\
\hline $\mathrm{RF}(\mathrm{IU} / \mathrm{mL})$ & $225.3 \pm 373.8$ \\
$\mathrm{ESR}(\mathrm{mm} / \mathrm{hr})$ & $40.9 \pm 31.9$
\end{tabular}


Lo et al., Afr J Tradit Complement Altern Med. (2013) 10(5):360-369 http://dx.doi.org/10.4314/ajtcam.v10i5.24

$\mathrm{CRP}(\mathrm{mg} / \mathrm{dl})$

Table 3: TCM pattern distribution in our study subjects

\begin{tabular}{ccc}
\hline & Patient number & $\%$ \\
\hline PT & 51 & 68.92 \\
SMT & 23 & 31.08 \\
SBSP & 64 & 86.49 \\
BSP & 69 & 93.24 \\
QSP & 40 & 54.05 \\
\hline
\end{tabular}

PT: purple tongue; SMT: stasis macule of tongue; SBSP: Sublingual blood stasis pattern; BSP: blood stasis pattern; QSP: qi stagnation pattern

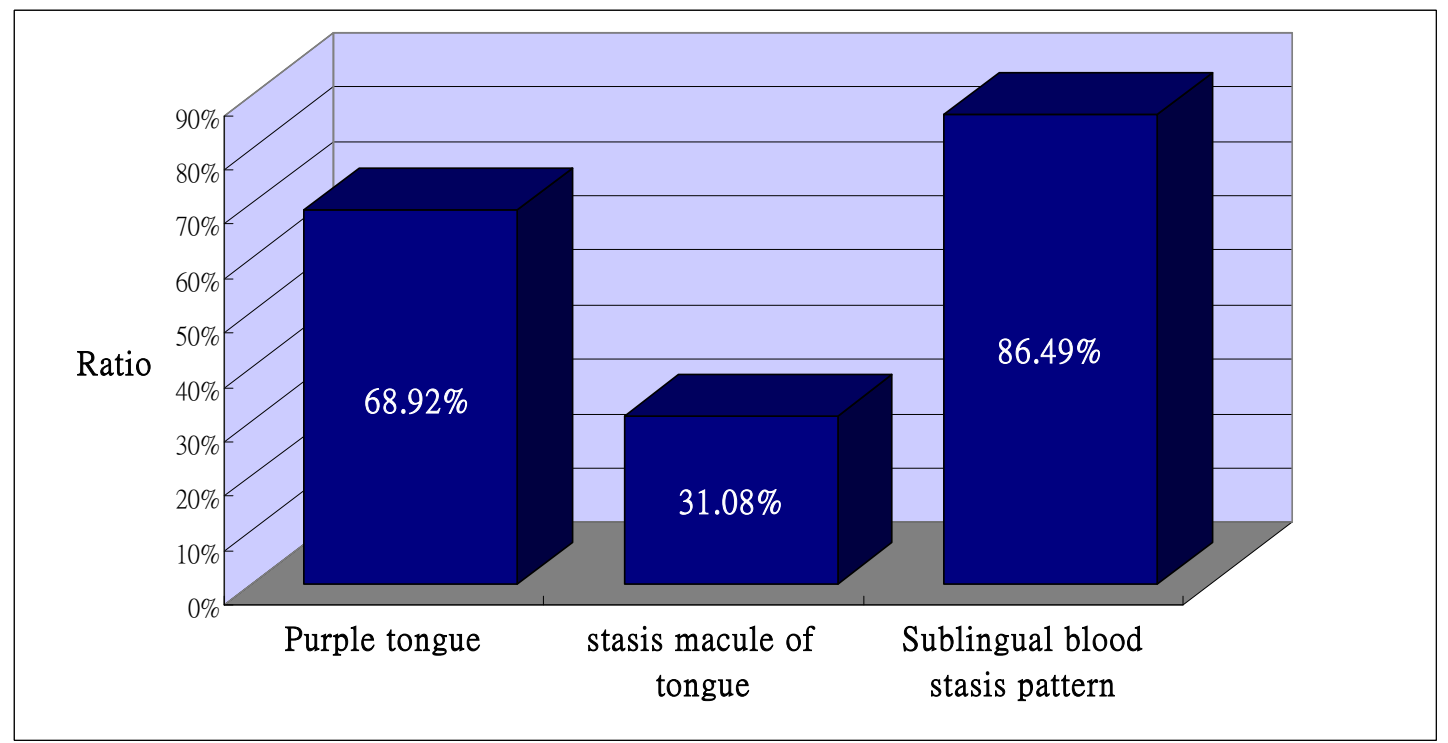

Figigure 1: Ratio of tongue characteristics in diagnosing blood stasis pattern in RA patients

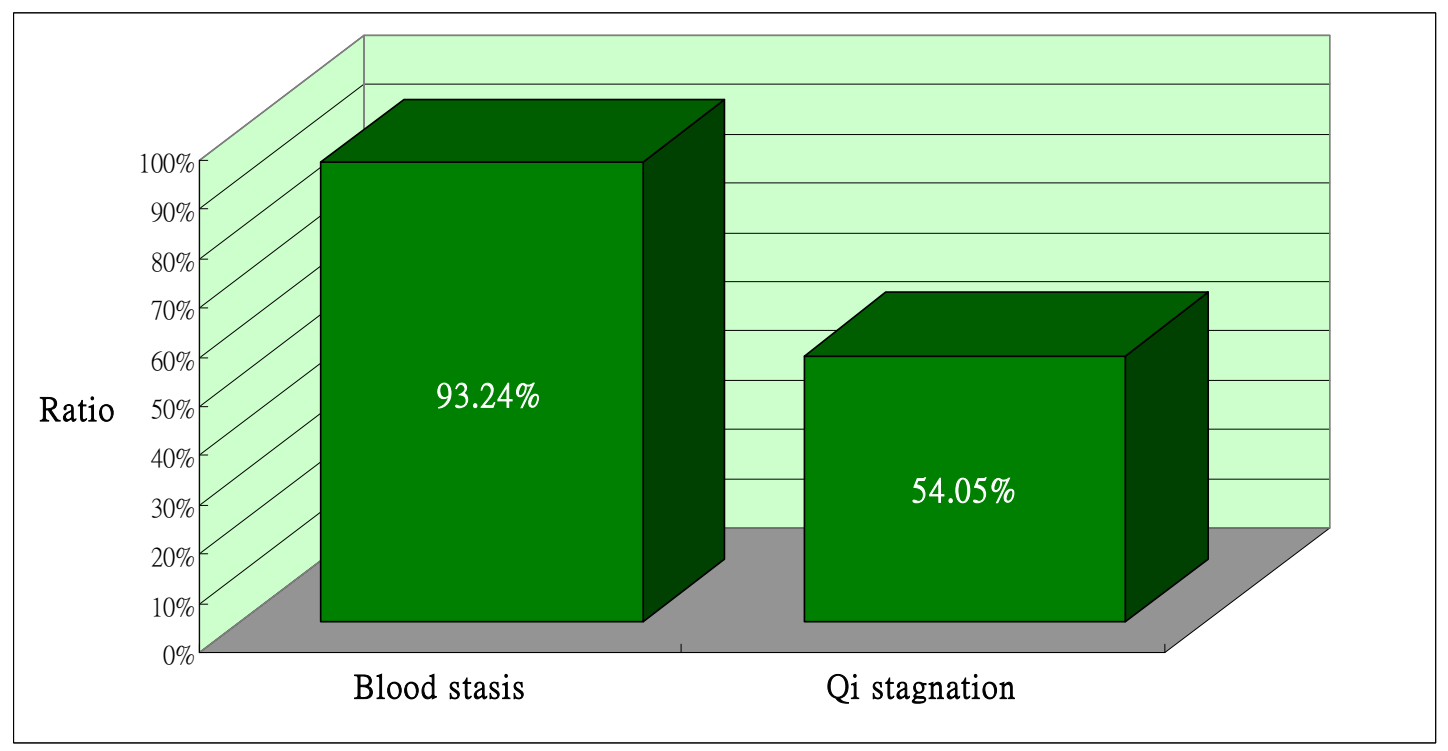

Figure 2: Ratio of blood stasis pattern and qi stagnation pattern in RA patients 


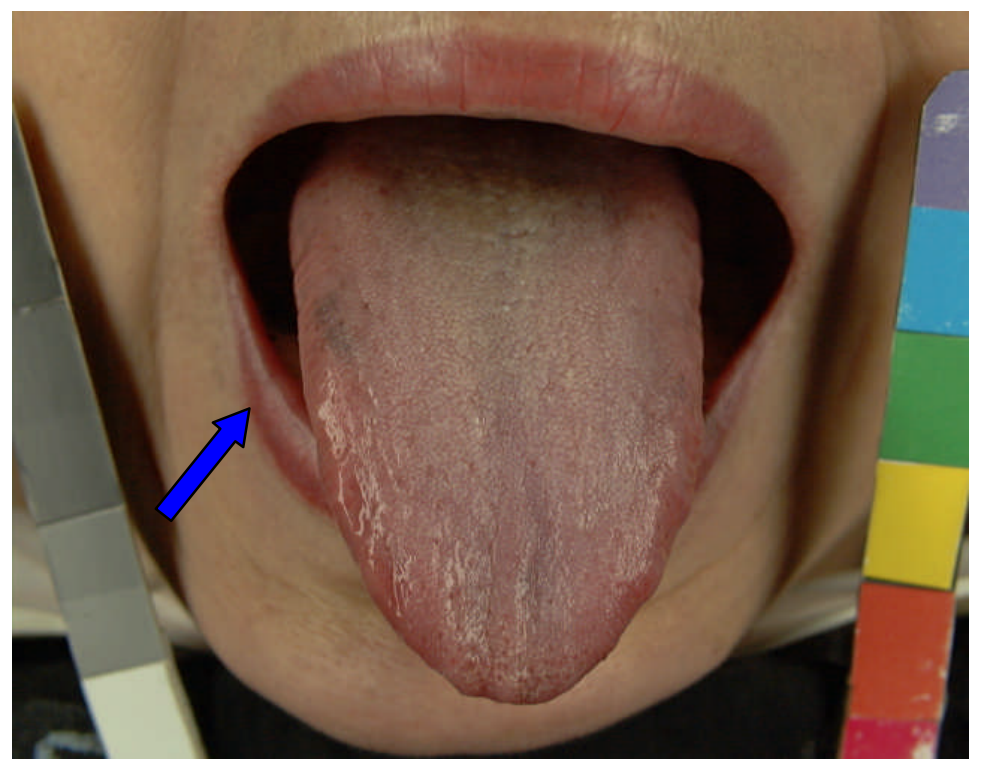

Figure 3: Stasis macule of tongue. The arrow points to a stasis macule of the tongue. In traditional Chinese medicine, this corresponds to the blood stasis pattern (BSP).

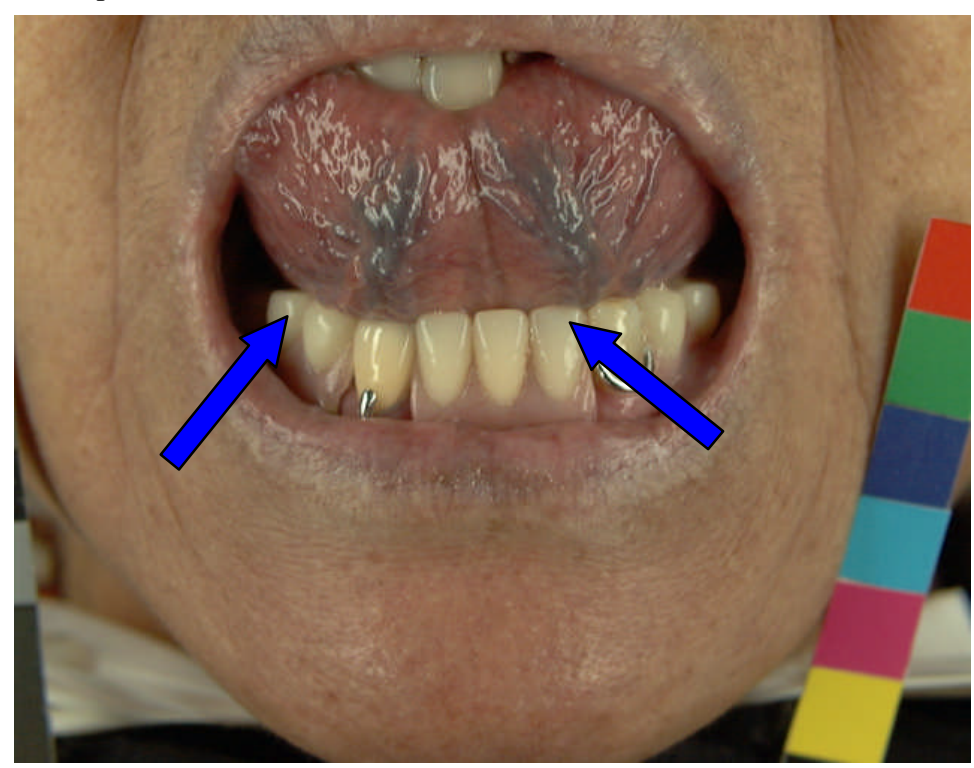

Figure 4: Sublingual blood stasis pattern. The arrows point to the sublingual vessel engorgement.

Table 4: Relation amongst RF, ESR and CRP and PT, SMT and SBSP

\begin{tabular}{cccc}
\hline & PT & SMT & SBSP \\
\hline RF & 0.513 & $0.007^{*}$ & 0.705 \\
ESR & 0.398 & 0.210 & 0.529 \\
CRP & 0.734 & 0.282 & 0.211
\end{tabular}


http://dx.doi.org/10.4314/ajtcam.v10i5.24

From Table 4, the RF index is statistically significantly correlated to the stasis macule of tongue. The other two indices, ESR and CRP, do not correlate to the three characteristics in Chinese tongue diagnosis. Besides, it is also interesting to study the relation between the three indices, RF, ESR and CRP, and the two patterns, Qi-stagnation pattern (QSP) and Blood Stasis pattern (BSP),that are used in Chinese medicine to determine the source of the disease.

Similarly, we also consider the generalised linear model since both QS and BS can be viewed as binary variables. The results are listed in Table 5. We find that only the relation between the RF and the QS pattern was mildly statistically significant.

Table 5: The correlation between laboratory data of RA patients' indices and TCM patterns

\begin{tabular}{ccc}
\hline & QSP & BSP \\
\hline RF & $0.059^{*}$ & 0.770 \\
ESR & 0.527 & 0.830 \\
CRP & 0.369 & 0.484 \\
\hline
\end{tabular}

P-values of generalized linear model $(\mathrm{N}=74) \quad *$ mild statistically significant

Table 6: Relation between RF and blood stasis pattern

\begin{tabular}{cccc}
\hline & & $\mathrm{RF}$ \\
\cline { 3 - 4 } & & $(+)$ & $(-)$ \\
\hline \multirow{2}{*}{ BSP } & $(+)$ & $49^{*}$ & 20 \\
& $(-)$ & 3 & 2 \\
\hline
\end{tabular}

* : patient number

We also analysed the relation between RF positive and blood stasis pattern. According Table 6, we find there are 49 patients of RF-blood stasis positive. This finding is consistent with that of Table 1, showing a high ratio of blood stasis pattern in RA patients.

\section{Discussion}

The present study is a preliminary research on the relation of tongue diagnosis of TCM and RA. The result of this research shows significant correlation between RF and stasis macule of tongue. Although purple tongue and sublingual blood stasis pattern also indicated blood stasis, it remains unclear whether only stasis macules of tongue and RF have strong correlation.

Rheumatoid factors (RF) are autoantibodies that react with the Fc portion of IgG. It is found in a variety of conditions such as immune system disorders, infection disease, or malignancies. About $80 \%$ of RA patients are seropositive for RF. (Cush et al. 2005) Although high level of RFs could not correlate with severe RA, patients with high level of RF may have severe extra-articular manifestations, such as rheumatoid nodules and vasculitis. (Harrison et al. 2006) Besides, some studies reported that RF was an indicator for prognosis of RA. (Mottonen et al. 1998; van Zeben et al. 1992) Patients with persistent positive RF had more radiological abnormalities, worse functional ability and disease outcome. (Mottonen et al. 1998)

Stasis macule of tongue is an important feature to diagnose blood stasis which may be caused by stagnation of qi, deficiency of qi or blood, trauma, or cold. It also occurs after long-term chronic disease. In TCM theory, blood stasis could 
predict the outcome of a disease. When a patient has a long-term, chronic disease, there might be some symptoms of blood stasis such as pain in a fixed location, stiffness at limbs, and clubbing of fingers and toes. (Neeb et al. 2007) Patients with blood stasis may also have poor prognosis of disease. In the beginning of this article, we introduced "Impediment disease" which symptoms were similar to RA. Blood stasis in Impediment disease might indicate poor prognosis and severe disease. Comparing blood stasis and RF, they were both indicators which could predict disease with similar signs and symptoms. Besides, RF brought out immune complexes in articular and extra-articular areas in some studies. One of its extra-articular manifestations is rheumatoid vasculitis. It may be caused from joint erosions, long-standing disease and high titiers of RF in serum and usually represented distal arthritis, cutaneous ulceration and palpable purpura. (Firestein et al. 2009) These symptoms were similar to symptoms of blood stasis. This may be the reason why there was significant correlation between $\mathrm{RF}$ and stasis macule of tongue. We also noted that qi stagnation pattern was mild statistically significant with $\mathrm{RF}(\mathrm{P}=0.059)$. According to TCM theory, Qi stagnation is a disorder in any organ or channel which affects the flow of qi and could lead to blood stasis. (Wiseman 1995; Yan 2004) This may explain that qi stagnation was mild statistically with RF. In this study, interand intra-agreement of physicians on tongue diagnosis is another important issue. Some previous researches reported low level of agreement because of inadequate operational definition of tongue characteristics.(Kim et al. 2008) But the agreement could improve after training. (Zhang et al. 2008) We demonstrated a test in order to study the agreement on tongue diagnosis among physicians. Thirty patients are grouped into three categories, namely, PT, SMT and SBSP. These 30 patients were deliberately chosen to be evenly distributed into each category. In this study, three TCM physicians were asked to judge if the patients in the three groups have apparent PT, SMT or SBSP. According to the rating data, we find all ratings agree perfectly no matter what agreement analysis is conducted.

A new approach, based on the TCM tongue diagnosis, is identified to differentiate RA from impediment disease. The tongue features extracted are compared with those obtained through Western medicine and the correlation between these two is investigated. The result demonstrates that blood stasis has significant correlation with RF, suggesting that the diagnosis of RA through relevant indicators of tongue diagnosis is promising. Nonetheless, further study is required to pinpoint the power of tongue diagnosis and replace routine lab tests with tongue diagnosis. The exact mechanism why blood stasis has significant relation with RF was still unknown. From previous studies in TCM and Western medicine, we guess that it may be because RF could induce vasculitis which effects circulation. The next phase of the study is to find the exact mechanism between blood stasis of TCM and RF, and enhance the reliability and validity of tongue diagnosis.

The severity of blood stasis of tongue diagnosis also was not clarified in this study. This is the other issue we need to work on for TCM doctors to predict the prognosis of RA through blood stasis of tongue diagnosis. In the future, we will do more research to connect TCM with Western medicine through tongue diagnosis or other diagnostic methods of TCM.

\section{Conclusion}

It is concluded that RF may associate closely with blood stasis pattern in TCM theory, and tongue diagnosis is helpful in detecting blood stasis of RA and could serve as a feasible predictor of the prognosis of RA. 
http://dx.doi.org/10.4314/ajtcam.v10i5.24

\section{References}

1. Basak, P., \& Jesmajian, S. (2010). Images in clinical medicine: A smooth patch on the tongue. N Engl J Med, 363, 1949

2. Choy, E.H., \& Panayi, G.S. (2001). Cytokine pathways and joint inflammation in rheumatoid arthritis. $N$ Engl J Med, $344,907-916$

3. Crowson, C.S., Rahman, M.U., \& Matteson, E.L. (2009). Which measure of inflammation to use? A comparison of erythrocyte sedimentation rate and C-reactive protein measurements from randomized clinical trials of golimumab in rheumatoid arthritis. J Rheumatol, 36, 1606-1610

4. Cush, J.J., Kavanaugh, A., \& Stein, C.M. (2005). Rheumatology : diagnosis and therapeutics. Philadephia: Lippincott, Williams \& Wilkins

5. Cylwik, B., Chrostek, L., Gindzienska-Sieskiewicz, E., Sierakowski, S., \& Szmitkowski, M. (2010). Relationship between serum acute-phase proteins and high disease activity in patients with rheumatoid arthritis. Adv Med Sci, 55, 80-85

6. Donald, F., \& Ward, M.M. (1998). Evaluative laboratory testing practices of United States rheumatologists. Arthritis Rheum, 41, 725-729

7. Firestein, G.S., Kelley, W.N., \& LLC, M.D.C. (2009). Kelley's textbook of rheumatology. Philadelphia, PA: Saunders/Elsevier

8. Fleming, A., Benn, R.T., Corbett, M., \& Wood, P.H. (1976). Early rheumatoid disease. II. Patterns of joint involvement. Ann Rheum Dis, 35, 361-364

9. Harrison, T.R., Fauci, A.S., \& Langford, C.A. (2006). Harrison's rheumatology. New York: McGraw-Hill

10. Kim, M., Cobbin, D., \& Zaslawski, C. (2008). Traditional Chinese medicine tongue inspection: an examination of the inter- and intrapractitioner reliability for specific tongue characteristics. J Altern Complement Med, 14, 527-536

11. Lee, D.M., \& Weinblatt, M.E. (2001). Rheumatoid arthritis. Lancet, 358, 903-911

12. Lichtenstein, M.J., \& Pincus, T. (1991). Rheumatoid arthritis identified in population based cross sectional studies: low prevalence of rheumatoid factor. J Rheumatol, 18, 989-993

13. Liu, C.A. (2005). Discussion of "Bi" in nei jin. Jiangxi Journal of Traditional Chinese Medicine, 36, 4

14. MacGregor, A.J. (1995). Classification criteria for rheumatoid arthritis. Baillieres Clin Rheumatol, 9, 287-304

15. Maciocia, G. (2004). Diagnosis in Chinese medicine : a comprehensive guide. Edinburgh; New York: Churchill Livingstone

16. Molenaar, E.T., Voskuyl, A.E., Familian, A., van Mierlo, G.J., Dijkmans, B.A., \& Hack, C.E. (2001). Complement activation in patients with rheumatoid arthritis mediated in part by C-reactive protein. Arthritis Rheum, 44, 997-1002

17. Moll, J.M., \& Wright, V. (1973). Psoriatic arthritis. Semin Arthritis Rheum, 3, 55-78

18. Mottonen, T., Paimela, L., Leirisalo-Repo, M., Kautiainen, H., Ilonen, J., \& Hannonen, P. (1998). Only high disease activity and positive rheumatoid factor indicate poor prognosis in patients with early rheumatoid arthritis treated with "sawtooth" strategy. Ann Rheum Dis, 57, 533-539

19. Neeb, G.R., Beer, M., Kaiser, J., \& Qing-ren, W. (2007). Blood stasis : China's classical concept in modern medicine, including a translation of the seminal work of Wang Qing-Ren 'Corrections of mistakes in the medical world'. Edinburgh: Churchill Livingstone Elsevier 
20. Negrato, C.A., \& Tarzia, O. (2010). Buccal alterations in diabetes mellitus. Diabetol Metab Syndr, 2,3

21. Otterness, I.G. (1994). The value of C-reactive protein measurement in rheumatoid arthritis. Semin Arthritis Rheum, 24, 91-104

22. Schnorrenberger, C.C., \& Schnorrenberger, B. (2005). Pocket atlas of tongue diagnosis : with Chinese therapy guidelines for acupuncture, herbs, and nutrition. Stuttgart; New York: Thieme

23. Shmerling, R.H., \& Delbanco, T.L. (1991). The rheumatoid factor: an analysis of clinical utility. Am J Med, 91 , $528-534$

24. Sionneau, P., \& Gang, L. (1998). Diseases of the neck, shoulders, back, and limbs. Boulder, Colo.: Blue Poppy Press

25. Toivanen, A., \& Toivanen, P. (2004). Reactive arthritis. Best Pract Res Clin Rheumatol, 18, 689-703

26. Unschuld, P.U. (2003). Huang Di nei jing su wen : nature, knowledge, imagery in an ancient Chinese medical text :

with an appendix, The doctrine of the five periods and six qi in the Huang Di nei jing su wen. Berkeley; Los Angeles; London: University of California Press

27. van Zeben, D., Hazes, J.M., Zwinderman, A.H., Cats, A., van der Voort, E.A., \& Breedveld, F.C. (1992). Clinical significance of rheumatoid factors in early rheumatoid arthritis: results of a follow up study. Ann Rheum Dis, 51, 1029-1035

28. Wiseman, N. (1995). Fundamentals of Chinese medicine : Zhong Yi Xué Ji Chu. Brookline, Mass.: Paradigm Publications

29. World Health Organization. Regional Office for the Western, P. (2007). WHO international standard terminologies on traditional medicine in the Western Pacific Region. [Manila, Philippines]: World Health Organization, Western Pacific Region

30. Yan, D. (2004). Aging \& blood stasis : a new TCM approach to geriatrics. Boulder, Colo.: Blue Poppy Press

31. Zhang, G.G., Singh, B., Lee, W., Handwerger, B., Lao, L., \& Berman, B. (2008). Improvement of agreement in TCM diagnosis among TCM practitioners for persons with the conventional diagnosis of rheumatoid arthritis: effect of training. $J$ Altern Complement Med, 14, 381-386 